\title{
Associations of breast milk adiponectin, leptin, insulin and ghrelin with maternal characteristics and early infant growth: a longitudinal study
}

\author{
Xinting $\mathrm{Yu}^{1,2,3}$, Shi Song Rong ${ }^{4}$, Xiujing Sun ${ }^{1}$, Guofang Ding ${ }^{1}$, Weilin Wan ${ }^{1}$, Liying Zou ${ }^{5}$, \\ Shaowen $\mathrm{Wu}^{5}$, Ming $\mathrm{Li}^{2 *}$ and Danhua Wang ${ }^{1 *}$ \\ ${ }^{1}$ Department of Pediatrics, Peking Union Medical College Hospital, Chinese Academy of Medical Sciences and Peking Union \\ Medical College, Beijing, People's Republic of China, 100730 \\ ${ }^{2}$ Key Laboratory of Endocrinology, Department of Endocrinology, National Health and Family Planning Commission, Peking \\ Union Medical College Hospital, Peking Union Medical College and Chinese Academy of Medical Science, Beijing, People's \\ Republic of China, 100730 \\ ${ }^{3}$ Department of Nutrition, Harvard T.H. Chan School of Public Health, Boston, MA O2115, USA \\ ${ }^{4}$ Department of Ophthalmology, Massachusetts Eye and Ear, Harvard Medical School, Boston, MA O2114, USA \\ ${ }^{5}$ Department of Obstetrics, Beijing Obstetrics and Gynecology Hospital, Beijing, People's Republic of China, 100010
}

(Submitted 30 March 2018 - Final revision received 24 August 2018 - Accepted 12 September 2018)

\section{Abstract}

Breast milk (BM) hormones have been hypothesised as a nutritional link between maternal and infant metabolic health. This study aimed to evaluate hormone concentrations in BM of women with and without gestational diabetes mellitus (GDM), and the relationship between maternal factors, BM hormones and infant growth. We studied ninety-six nulliparous women with ( $n$ 48) and without GDM and their exclusively breastfed term singletons. Women with GDM received dietary therapy or insulin injection for euglycaemia during pregnancy. Hormone concentrations in BM, maternal BMI and infant growth were longitudinally evaluated on postnatal days 3, 42 and 90. Mothers with GDM had decreased concentrations of adiponectin $\left(P_{\text {colostrum }}<0.001 ; P_{\text {mature-milk }}=0.009\right)$ and ghrelin $\left(P_{\text {colostrum }}=0.011 ; P_{\text {mature-milk }}<0 \cdot 001\right)$ and increased concentration of insulin in BM $\left(P_{\text {colostrum }}=0.047 ; P_{\text {mature-milk }}=0.021\right)$. Maternal BMI was positively associated with adiponectin $(\beta=0.06 ; 95 \%$ CI $0.02,0 \cdot 1 ; P=0.001)$, leptin $(\beta=0 \cdot 16 ; 95 \%$ CI $0 \cdot 12,0 \cdot 2 ; P<0 \cdot 001)$ and insulin concentrations $(\beta=0 \cdot 06 ; 95 \%$ CI $0.02,0 \cdot 1$; $P<0 \cdot 001)$, and inversely associated with ghrelin concentration in $\mathrm{BM}(\beta=-0.08 ; 95 \% \mathrm{CI}-0 \cdot 1,-0 \cdot 06 ; P<0 \cdot 001)$. Among the four hormones, adiponectin was inversely associated with infant growth in both the GDM $\left(\beta_{\text {weight-for-height }}=-2 \cdot 49 ; 95 \%\right.$ CI $-3 \cdot 83,-1 \cdot 15 ; P<0 \cdot 001$; $\left.\beta_{\text {head-circumference }}=-0 \cdot 39 ; 95 \% \mathrm{CI}-0.65,-0 \cdot 13 ; P=0.003\right)$ and healthy groups $\left(\beta_{\text {weight } \text { for-height }}=-1 \cdot 42 ; 95 \% \mathrm{CI}-2 \cdot 38,-0 \cdot 46 ; P=0 \cdot 003\right.$; $\beta_{\text {head-circumference }}=-0 \cdot 15 ; 95 \%$ CI $\left.-0.27,-0.03 ; P=0 \cdot 007\right)$. Maternal BMI and GDM are important determinants of BM hormone concentrations. Milk-borne adiponectin is determined by maternal metabolic status and plays an independent down-regulating role in early infant growth.

Key words: Breast milk: Hormones: Infant growth: Gestational diabetes mellitus

In parallel with the increasing trend of gestational diabetes mellitus $(\mathrm{GDM})^{(1)}$, the prevalence of childhood obesity has been increasing at an alarming rate of up to $18 \%$ worldwide ${ }^{(2)}$. Mounting evidence indicates that children born to mothers with diabetes have a higher risk of high birth weight ${ }^{(3)}$, amplified adiposity in infancy ${ }^{(4)}$, later obesity ${ }^{(5)}$ and type 2 diabetes ${ }^{(6)}$. The growth of these children could be 'programed' during pregnancy by intra-uterine over-nutrition and follow adverse trajectories characterised by larger size and accelerated velocity of weight gain during infancy and childhood. Nutrition during infancy is an important modulator for energy balance throughout life ${ }^{(7)}$. Breast milk (BM) as the natural food for infant has been recommended widely in general population for its protective effects against obesity ${ }^{(8)}$ and type 2 diabetes $^{(9)}$ in later life. However, the effect of breast-feeding on the future obesity of the children born to mothers with GDM remains uncertain. Studies of mothers with diabetes showed contradictory results, including increasing the risk ${ }^{(10)}$, no effects on the risk $^{(11)}$, or lowering increased adiposity associated with exposure to diabetes in utero $^{(12)}$.

The favourable energy balance and growth of breastfed infants have been suggested to be derived in part from the hormones in $\mathrm{BM}^{(13)}$. Hormones are stable in $\mathrm{BM}$ and could be absorbed through the receptors in human gastrointestinal

\footnotetext{
Abbreviations: BM, breast milk; GDM, gestational diabetes mellitus.
}

* Corresponding authors: M. Li, fax +86 10 69155073, email liming@pumch.cn; D. Wang, fax +86 1069156271 , email danhuawang10@gmail.com 
tract $^{(14-17)}$. Some bioactive BM hormones are reported to link maternal metabolic status with metabolic health of offspring. For instance, adiponectin and leptin concentrations in BM are associated with maternal $\mathrm{BMI}^{(18-21)}$ and could be altered by $\mathrm{GDM}^{(22,23)}$. Breastfed infants exposed to higher concentration of adiponectin in BM have a lower weight gain during the first 6 months ${ }^{(24)}$. However, the effects of maternal factors on adiponectin and leptin were inconsistent among studies, and the inconclusive results regarding insulin and ghrelin render further confirmation $^{(25)}$. The association of BM hormone with infant growth was also inconclusive in previous studies using different anthropometric measurements, such as weight gain ${ }^{(18)}$, body composition $^{(20)}$, BMI $^{(21,26-29)}$ or weight-for-height ${ }^{(24,29,30)}$. Moreover, few studies examined the links in women with GDM and their infants.

In this study, we recruited women with $\mathrm{GDM}^{(31)}$, healthy women and their exclusively breastfed infants for longitudinal follow-ups on days 3,42 and 90. We aimed to evaluate the adiponectin, leptin, insulin and ghrelin concentrations in BM of women with GDM and the relationship between maternal factors, BM hormones and early infant growth.

\section{Methods}

The study protocol was approved by the institutional review boards at Peking Union Medical College Hospital and Beijing Obstetrics and Gynecology Hospital. Signed informed consent was obtained from all the participating families. Trial identifcation number and URL: NCT03145649 https://clinicaltrials.gov/ show/NCT03145649.

\section{Subjects}

Nulliparous women with GDM and healthy women who intended to exclusively breastfeed their singletons were recruited consecutively from the obstetric wards at Peking Union Medical College Hospital and Beijing Obstetrics and
Gynecology Hospital during the 37th gestational week. The exclusion criteria were: pre-pregnancy diabetes, fetal anomaly, gestational hypertension, pre-eclampsia, fetal growth restriction, ruptured membranes, postpartum glucose abnormalities (see below) and introduction of formula feeding during the follow-ups. Women with plasma glucose $>7.8 \mathrm{mmol} / \mathrm{l}$ in the $1 \mathrm{~h}$ $50 \mathrm{~g}$ glucose load test (GLT) during the 24th to 28th gestational week underwent a $3 \mathrm{~h} 100 \mathrm{~g}$ diagnostic oral glucose tolerance test (OGTT) following a $12 \mathrm{~h}$ overnight fast. GDM was diagnosed if two or more plasma glucose reads equaled or exceeded the threshold according to Carpenter/Coustan diagnostic criteria ${ }^{(32)}$. All subjects diagnosed with GDM initially received dietary therapies to ensure euglycaemia, adequate nutrition and appropriate weight gain. Those who did not achieve glycaemic targets (3.3-5.6 mmol/1 at fasting, $3.3-5.8 \mathrm{mmol} / 1$ pre-prandially, $4.4-$ $6.7 \mathrm{mmol} / \mathrm{l} 2 \mathrm{~h}$ post-prandially and $4.4-6.7 \mathrm{mmol} / \mathrm{l}$ at night) in 2 weeks were given insulin via injection. As the close link between BM macronutrients and the maternal glucose metabolic status could potentially bias our evaluation of the association between BM hormones and infant growth, we excluded women with postpartum glucose abnormalities, that is, impaired glucose tolerance (IGT) with the $2 \mathrm{~h}$ plasma glucose between 7.8 and $11.0 \mathrm{mmol} / \mathrm{l}$ and type 2 diabetes with the $2 \mathrm{~h}$ plasma glucose $\geq 11.1 \mathrm{mmol} / \mathrm{l}$ in $75 \mathrm{~g}$ OGTT on day 42 (Fig. 1).

\section{Anthropometric measurements}

Obstetric data including glycaemic tests, gestational age and mode of delivery were collected from medical records. Prepregnancy weight was self-reported. Height was measured twice to the nearest $0 \cdot 1 \mathrm{~cm}$ with a wall-mounted stadiometer. Weight was measured twice to the nearest $0 \cdot 1 \mathrm{~kg}$ with a medical balance scale before delivery, on postpartum days 42 and 90. Infant weight, length and head circumference were measured at birth, on days 42 and 90. The infants were weighed twice in nude using a precision scale (Seca). Body length and head circumference were measured twice to the nearest $0 \cdot 1 \mathrm{~cm}$ with a length board and non-stretchable measuring tapes (Seca). We

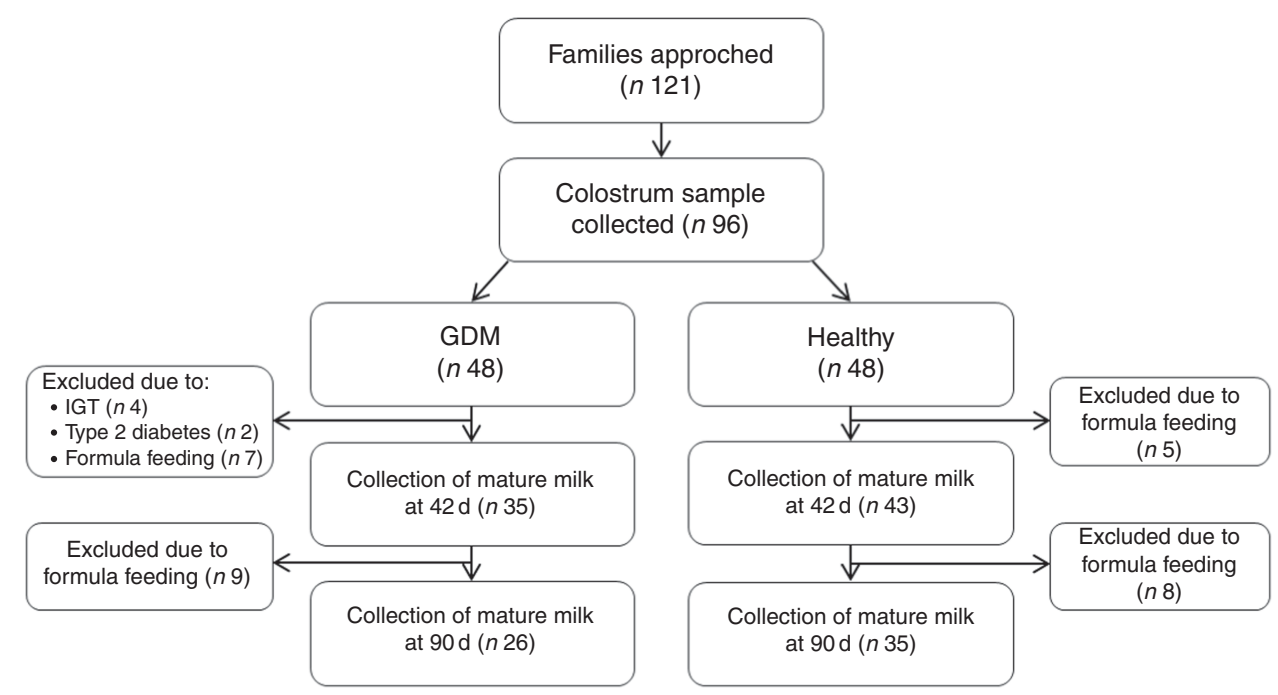

Fig. 1. Diagram of participant and follow-up flow. GDM, gestational diabetes mellitus; IGT, impaired glucose tolerance. 
averaged the two readings for data analysis.

\section{Milk sample collection, processing and laboratory tests}

Colostrum samples were collected between 08.00 and 09.00 hours before infant feeding on the 3rd day after delivery. Mature milk, including both foremilk and hindmilk, was delivered and collected from one breast before infant feeding using an electric pump (Medela) between 14.00 and 16.00 hours on days 42 and 90 in outpatient clinics. Milk samples were frozen immediately in sterilised plastic tubes at $-80^{\circ} \mathrm{C}$. Before quantifying the hormones in $\mathrm{BM}$, the samples were thawed at $4^{\circ} \mathrm{C}$, sonicated and centrifuged. Sonication was performed at $50 \mathrm{~W}$ for three bursts with 10-s intervals, and centrifuged at $100000 \mathrm{~g}$ for $1 \mathrm{~h}$ at $4^{\circ} \mathrm{C}$ (Braun-sonic Sonicator; B. Braun). The supernatant fat was discarded and the skim milk was used for quantifying adiponectin, leptin, insulin and ghrelin by ELISA at the Key Laboratory of Endocrinology, Peking Union Medical College Hospital. The intra- and inter-assay CV were $<5.4$ and $<8.5 \%$ for adiponectin, and $<7.4$ and $<9.3 \%$ for leptin, respectively. The cross-reactivity to proinsulin of the insulin assay was not significant $(<0.05 \%)$. The sensitivity of insulin assay was $0.5 \mathrm{mU} / \mathrm{l}$, and the intra- and inter-assay $\mathrm{CV}$ were $<4.1$ and $<9.0 \%$. Total ghrelin was tested using the total human ghrelin ELISA kit (Millipore). The intra- and inter-assay CV for the ghrelin assay were $<1.9$ and $<7.7 \%$.

\section{Statistical analysis}

According to the study on colostrum ghrelin concentration in women with and without $\mathrm{GDM}^{(33)}$, six mother-infant dyads at each group were needed to achieve the statistical power of $84 \%$ and the two-sided significance level of $0 \cdot 05^{(34)}$. According to the baseline adiponectin level and infant weight-for-height in another two cohorts examining the association between BM adiponectin and infant growth ${ }^{(24)}$, a sample size of seventy-two (using data from Cincinnati group) or seventy-five (using data from Maxico group) mother-infant dyads was able to achieve a statistical power of $80 \%{ }^{(35)}$. We had ninety-six mother-infant dyads at the baseline and seventy-eight on day 42 , which was an acceptable sample size for detecting the association between $\mathrm{BM}$ adiponectin concentration and infant weight-for-height. The differences in the demographic characteristics and hormone concentrations between GDM and healthy groups were evaluated using independent sample $t$ test and Mann-Whitney $U$ test for continuous variables, and using $\chi^{2}$ test for discrete variables. The association of maternal and obstetrical factors (i.e. pre-pregnancy BMI, maternal BMI during lactation, GDM, plasma glucose during pregnancy, gestational weight gain, gestational age and delivery mode) with the hormone concentrations was tested using generalised estimating equation (GEE), a semi-parametric analysis using all the longitudinal data points to estimate the overall average effects of maternal or obstetrical factors (e.g. BMI) on the hormone levels in BM. The association of the overall hormone concentrations in BM with infant weight-for-height gain and head circumference was also analysed using GEE. The statistical analysis was conducted using SPSS version 20.0 (SPSS Inc.). $\alpha$ was set to 0.05 for two- sided tests if otherwise mentioned. To be more conservative with our exploratory analysis, we used Bonferroni correction to control for potentially inflated type I error $(\alpha)$ in the regression analyses.

\section{Results}

In all, ninety-six of 121 eligible women agreed to participate and successfully delivered colostrum. Among them, forty-eight women were with GDM and forty-eight were healthy (Fig. 1). In the GDM group, twenty-two dyads dropped out due to the introduction of formula feeding ( $n$ 16), IGT ( $n$ 4) and type 2 diabetes $(n 2)$. A total of thirteen healthy mothers dropped out due to the introduction of formula feeding (Fig. 1).

The plasma glucose of women with GDM at fasting, 1, 2 and $3 \mathrm{~h}$ in OGTT were 5.75 (SD 0.98), 10.96 (SD 1.51), 9.78 (SD 1.47 ) and $7 \cdot 14$ (SD 1.95) $\mathrm{mmol} / \mathrm{l}$, respectively (Table 1 ). As expected, women with GDM had higher plasma glucose at $1 \mathrm{~h}$ in the $50 \mathrm{~g}$ GLT during pregnancy compared with healthy women (11.49 (sD 2.42) v. 6.82 (sD 1.29), $P<0.001$; Table 1). We tested glycosylated $\mathrm{Hb}$ for women with a diagnosis of GDM during the 24 th to 28 th gestational weeks. The median glycosylated $\mathrm{Hb}$ level was $6.20 \%(44 \mathrm{mmol} / \mathrm{mol}$; ranged from $5.0 \%$ (31 $\mathrm{mmol} / \mathrm{mol})$ to $10.0 \%(86 \mathrm{mmol} / \mathrm{mol})$; data not shown). In all, seventeen mothers with GDM received insulin injections. All mothers with GDM had favourable blood glucose control during pregnancy.

The two maternal groups were comparable in age, gestational age and delivery mode $(P>0 \cdot 05$; Table 1$)$. Women with GDM, particularly those who received insulin injections, had higher BMI $(P \leq 0.012)$ but less gestational weight gain $(P=0.009)$ than healthy women (online Supplementary Table S1). We did not find significant differences between infants born to GDM and healthy women, regarding their weight, height, weight-for-height and head circumference at birth, on days 42 and $90(P>0 \cdot 05$; Table 1$)$.

\section{Breast milk hormone concentrations and maternal factors}

We collected colostrum samples on day 3, and mature milk samples on days 42 and 90 (Fig. 1). When compared with healthy women, women with GDM had lower concentrations of adiponectin and ghrelin in colostrum on day 3 ( $P_{\text {adiponectin }}$ $<0.001$ and $\left.P_{\text {ghrelin }}=0.011\right)$ and mature milk on day 90 $\left(P_{\text {adiponectin }}=0.009\right.$ and $P_{\text {ghrelin }}<0.001$; Table 2). Women with GDM had higher concentration of insulin in colostrum $(P=0.047)$ and mature milk $(P=0.021$; Table 2$)$, especially in women who received insulin injections $\left(P_{\text {colostrum }}=0.049\right.$ and $P_{\text {mature milk }}<0.001$; online Supplementary Table S2). The leptin concentration was not statistically different between women with GDM and healthy women $(P>0.05$; Table 2$)$. All the four hormones were not significantly different between the two groups on day 42 ( $P>0.05$; Table 2$)$. Lactation time also played an important role in adiponectin, leptin and ghrelin levels in BM. Adiponectin and leptin concentration decreased across time and ghrelin concentration was highest at day 42 (Table 2). 
Table 1. Characteristics of mothers and infants

(Mean values and standard deviations; numbers and percentages)

\begin{tabular}{|c|c|c|c|c|c|}
\hline & \multicolumn{2}{|c|}{ GDM group $(n 48)^{*}$} & \multicolumn{2}{|c|}{ Healthy group $(n 48)^{\star}$} & \multirow[b]{2}{*}{$P \dagger$} \\
\hline & Mean & SD & Mean & SD & \\
\hline \multicolumn{6}{|l|}{ Mothers } \\
\hline Age (years) & $32 \cdot 15$ & 3.84 & 32.04 & 3.58 & 0.891 \\
\hline 1-h $50 \mathrm{~g} \mathrm{GLT}(\mathrm{mmol} / \mathrm{l})$ & 11.49 & 2.42 & 6.82 & 1.29 & $<0.001$ \\
\hline \multicolumn{6}{|l|}{ OGTT } \\
\hline Fasting & 5.75 & 0.98 & 4.40 & 0.25 & $<0.001$ \\
\hline $1 \mathrm{~h}$ & $10 \cdot 96$ & 1.51 & 7.76 & 1.48 & $<0.001$ \\
\hline $2 \mathrm{~h}$ & 9.78 & 1.47 & 6.54 & $1 \cdot 16$ & $<0.001$ \\
\hline $3 \mathrm{~h}$ & $7 \cdot 14$ & 1.95 & $5 \cdot 61$ & 1.15 & 0.041 \\
\hline Gestational weight gain $(\mathrm{kg})$ & $13 \cdot 56$ & 6.05 & $17 \cdot 06$ & 5.03 & 0.004 \\
\hline Gestational age at delivery (weeks) & 38.56 & 0.71 & 38.92 & 0.99 & 0.066 \\
\hline \multicolumn{6}{|l|}{ Delivery mode } \\
\hline Vaginal delivery & \multirow{2}{*}{\multicolumn{2}{|c|}{10}} & \multirow{2}{*}{\multicolumn{2}{|c|}{15}} & 0.245 \\
\hline$n$ & & & & & \\
\hline$\%$ & \multicolumn{2}{|c|}{21} & \multicolumn{2}{|c|}{31} & \\
\hline \multicolumn{6}{|l|}{ Caesarean section delivery } \\
\hline$n$ & \multicolumn{2}{|c|}{38} & \multicolumn{2}{|c|}{33} & \\
\hline$\%$ & \multicolumn{2}{|c|}{79} & \multicolumn{2}{|c|}{69} & \\
\hline \multicolumn{6}{|l|}{$\operatorname{BMI}\left(\mathrm{kg} / \mathrm{m}^{2}\right)$} \\
\hline Pre-pregnancy & $22 \cdot 87$ & $3 \cdot 20$ & $20 \cdot 71$ & $3 \cdot 27$ & 0.002 \\
\hline Pre-delivery & 27.97 & 3.56 & 27.22 & 3.31 & 0.293 \\
\hline Day 42 & $24 \cdot 17$ & 3.34 & 23.42 & 3.49 & 0.339 \\
\hline Day 90 & 23.97 & $3 \cdot 30$ & 21.94 & 2.65 & 0.009 \\
\hline \multicolumn{6}{|l|}{ Infants } \\
\hline Boys & & & 0.679 \\
\hline$n$ & \multirow{2}{*}{\multicolumn{2}{|c|}{$\begin{array}{l}27 \\
56\end{array}$}} & & & \\
\hline$\%$ & & & \multicolumn{2}{|c|}{$\begin{array}{l}29 \\
60\end{array}$} & \\
\hline \multicolumn{6}{|l|}{ Weight $(g)$} \\
\hline Birth & 3468.85 & 425.87 & 3342.50 & $440 \cdot 36$ & 0.156 \\
\hline Day 42 & 4954.08 & 468.45 & 5157.09 & 549.91 & 0.076 \\
\hline Day 90 & $6672 \cdot 10$ & $577 \cdot 24$ & $6857 \cdot 18$ & $692 \cdot 20$ & 0.232 \\
\hline \multicolumn{6}{|l|}{ Height (cm) } \\
\hline Birth & $50 \cdot 13$ & 1.59 & 49.90 & 1.60 & 0.484 \\
\hline Day 42 & $56 \cdot 71$ & 1.86 & 57.07 & $2 \cdot 18$ & 0.425 \\
\hline Day 90 & $62 \cdot 60$ & $2 \cdot 18$ & 63.02 & 2.04 & 0.397 \\
\hline \multicolumn{6}{|l|}{ Weight-for-height ( $\mathrm{g} / \mathrm{cm})$} \\
\hline Birth & 69.07 & 6.88 & 66.85 & $7 \cdot 19$ & 0.126 \\
\hline Day 42 & $87 \cdot 27$ & $6 \cdot 51$ & $90 \cdot 35$ & 8.90 & 0.071 \\
\hline Day 90 & $106 \cdot 52$ & 7.57 & $108 \cdot 75$ & 9.81 & 0.297 \\
\hline Head circumference $(\mathrm{cm})$ & & & & & \\
\hline Birth & 34.64 & 1.06 & $34 \cdot 30$ & 1.03 & 0.156 \\
\hline Day 42 & $38 \cdot 23$ & 1.20 & 38.39 & 1.07 & 0.542 \\
\hline Day 90 & $40 \cdot 46$ & 0.94 & 40.68 & 0.97 & 0.335 \\
\hline
\end{tabular}

GDM, gestational diabetes mellitus; GLT, glucose loading test; OGTT, oral glucose tolerance test.

* The number of subjects at recruitment and first follow-up. The numbers of subjects at the second and third follow-ups are shown in Fig. 1.

$t t$ Tests for continuous variables and $x^{2}$ tests for categorical variables were used to evaluate the difference between GDM and healthy groups.

‡ All mothers with GDM and eight healthy mothers underwent OGTT during pregnancy.

We tested the association of maternal factors with overall hormone concentrations in BM using GEE. We found that adiponectin concentration was inversely associated with GDM $(P=0 \cdot 014)$, plasma glucose concentration at $1 \mathrm{~h}$ in the $50 \mathrm{~g}$ GLT during pregnancy $(P<0 \cdot 001)$, and caesarean section delivery $(P=0.029)$, while it was positively associated with maternal BMI during lactation $(P=0 \cdot 001)$ and gestational age $(P=0 \cdot 017$; Table 3). Leptin concentration was only positively associated with maternal body size, including pre-pregnancy BMI $(P=0.017)$ and maternal BMI during lactation $(P<0.001$; Table 3). Furthermore, we noticed that pre-pregnancy BMI, maternal BMI during lactation, GDM and plasma glucose concentration at $1 \mathrm{~h}$ in $50 \mathrm{~g}$ GLT during pregnancy were positively associated with insulin concentration $(P<0.001$ for pre-pregnancy $\mathrm{BMI}$, maternal BMI during lactation and GDM; $P=0.035$ for plasma glucose concentration), while inversely associated with ghrelin concentration in BM $(P=0.031$ for pre-pregnancy BMI, $P<0.001$ for maternal BMI during lactation and GDM, and $P=0.007$ for plasma glucose concentration; Table 3). However, some of the associations, such as GDM and gestational age with adiponectin, were insignificant with Bonferroni correction rendering further confirmation (Table 3). 
Table 2. Breast milk hormone concentrations over lactation (Medians and interquartile ranges (IQR))

\begin{tabular}{|c|c|c|c|c|c|c|}
\hline & \multicolumn{2}{|c|}{ GDM } & \multicolumn{2}{|c|}{ Healthy } & \multirow[b]{2}{*}{$P_{\text {group }}{ }^{*}$} & \multirow[b]{2}{*}{$P_{\text {time }} \dagger$} \\
\hline & Median & IQR & Median & IQR & & \\
\hline \multicolumn{7}{|c|}{ Adiponectin (ng/ml) } \\
\hline Day 3ł & $21 \cdot 74$ & $14 \cdot 77-56 \cdot 10$ & $65 \cdot 81$ & $29 \cdot 76-126.91$ & $<0.001$ & $0.001 \S$ \\
\hline Day 42\| & 11.89 & $8 \cdot 00-18 \cdot 37$ & $12 \cdot 22$ & 9.69-14.92 & 0.889 & \\
\hline Day 90ף & $11 \cdot 75$ & $8.53-13.91$ & $15 \cdot 31$ & $11.60-19.53$ & 0.009 & \\
\hline \multicolumn{7}{|c|}{ Leptin (ng/ml) } \\
\hline Day 3‡ & 1.28 & $0.87-2.63$ & 1.49 & $0.56-3.25$ & 0.774 & $<0.001^{\star \star}$ \\
\hline Day 42\| & 0.26 & $0.09-0.47$ & 0.21 & $0.09-0.51$ & 0.692 & \\
\hline Day 90ף & 0.20 & $0.12-0.47$ & 0.25 & $0.16-0.45$ & 0.539 & \\
\hline \multicolumn{7}{|c|}{ Insulin $(\mu \mathrm{U} / \mathrm{ml})$} \\
\hline Day 3‡ & $22 \cdot 80$ & $13.51-51 \cdot 25$ & $20 \cdot 41$ & $7 \cdot 68-31.38$ & 0.047 & 0.101 \\
\hline Day 42\| & $32 \cdot 36$ & $13 \cdot 06-58 \cdot 22$ & $28 \cdot 20$ & $17.97-40.05$ & 0.376 & \\
\hline Day 90ף & $40 \cdot 63$ & $22 \cdot 48-57 \cdot 17$ & $24 \cdot 61$ & $13 \cdot 40-31 \cdot 85$ & 0.021 & \\
\hline \multicolumn{7}{|c|}{ Ghrelin (pg/ml) } \\
\hline Day 3‡ & 124.43 & $89 \cdot 87-178 \cdot 76$ & $159 \cdot 36$ & $122 \cdot 62-234 \cdot 33$ & 0.011 & $0.004 \dagger \dagger$ \\
\hline Day 42\| & $338 \cdot 74$ & $189.98-432.95$ & 337.60 & $149.82-565.77$ & 0.795 & \\
\hline Day 90ף & $104 \cdot 62$ & $72 \cdot 72-154 \cdot 71$ & $210 \cdot 91$ & $147 \cdot 25-381 \cdot 88$ & $<0.001$ & \\
\hline
\end{tabular}

GDM, gestational diabetes mellitus.

* Mann-Whitney $U$ tests were used to compare the difference between GDM and healthy groups at each lactation time point.

$\dagger$ General linear model for repeated measurements with GDM/without GDM as between-subject factor was used to compare the hormone concentrations over time. Hormone concentrations were natural log transformed before entering the models.

$\ddagger n 48$ for GDM group and $n 48$ for healthy group at day 3 .

$\S$ In subsequent within-subject contrasts, adiponectin concentration was significantly different between day 3 and day 42 $(P=0.001)$.

II $n 35$ for GDM group and $n 43$ for healthy group at day 42 .

II $n 26$ for GDM group and $n 35$ for healthy group at day 90 .

${ }^{* *}$ In subsequent within-subject contrasts, leptin concentration was significantly different between days 3 and $42(P<0.001)$ and between days 3 and $90(P=0.002)$.

†† In subsequent within-subject contrasts, ghrelin concentration was significantly different between days 3 and $42(P<0.001)$ and between days 42 and $90(P=0.044)$.

Table 3. Associations between maternal factors and breast milk hormone concentrations $\dagger$

( $\beta$-Coefficients and $95 \%$ confidence intervals)

\begin{tabular}{|c|c|c|c|c|c|c|c|c|c|c|c|c|}
\hline & \multicolumn{3}{|c|}{ Adiponectin (ng/ml) } & \multicolumn{3}{|c|}{ Leptin (ng/ml) } & \multicolumn{3}{|c|}{ Insulin $(\mu \mathrm{U} / \mathrm{ml})$} & \multicolumn{3}{|c|}{ Ghrelin (pg/ml) } \\
\hline & $\beta$ & $95 \% \mathrm{Cl}$ & $P^{\star}$ & $\beta$ & $95 \% \mathrm{Cl}$ & $P^{\star}$ & $\beta$ & $95 \% \mathrm{Cl}$ & $P^{\star}$ & $\beta$ & $95 \% \mathrm{Cl}$ & $P^{\star}$ \\
\hline \multicolumn{13}{|l|}{ Maternal factors } \\
\hline Pre-pregnancy BMI & -0.03 & $-0.07,0.01$ & 0.305 & 0.06 & $0.02,0.1$ & 0.017 & 0.09 & $0.05,0.13$ & $<0.001$ & -0.05 & $-0.09,-0.01$ & 0.031 \\
\hline Maternal BMI during lactation & 0.06 & $0.02,0.1$ & 0.001 & 0.16 & $0.12,0.2$ & $<0.001$ & 0.06 & $0.02,0.1$ & $<0.001$ & -0.08 & $-0.1,-0.06$ & $<0.001$ \\
\hline GDM & -0.39 & $-0.71,-0.07$ & 0.014 & 0.01 & $-0.35,0.37$ & 0.961 & 0.5 & $0.22,0.78$ & $<0.001$ & -0.53 & $-0.75,-0.31$ & $<0.001$ \\
\hline $\begin{array}{l}\text { 1-h plasma glucose in } 50 \mathrm{~g} \mathrm{GLT} \\
(\mathrm{mmol} / \mathrm{l})\end{array}$ & -0.08 & $-0.12,-0.04$ & $<0.001$ & 0.01 & $-0.07,0.09$ & 0.823 & 0.06 & $0.01,0.12$ & 0.035 & -0.06 & $-0.1,-0.02$ & 0.007 \\
\hline Gestational weight gain (kg) & 0.01 & $-0.01,0.03$ & 0.746 & -0.02 & $-0.06,0.02$ & 0.375 & -0.02 & $-0.04,0.01$ & 0.211 & -0.01 & $-0.03,0.01$ & 0.501 \\
\hline \multicolumn{13}{|l|}{ Obstetrical factors } \\
\hline Gestational age (weeks) & 0.2 & $0.04,0.36$ & 0.017 & 0.09 & $-0.11,0.29$ & 0.381 & -0.02 & $-0 \cdot 2,0 \cdot 16$ & 0.788 & $0 \cdot 1$ & $-0.02,0.22$ & 0.082 \\
\hline $\begin{array}{l}\text { Delivery mode (Caesarean } \\
\text { section) }\end{array}$ & -0.38 & $-0.74,-0.02$ & 0.029 & $-0 \cdot 27$ & $-0.71,0.17$ & 0.227 & -0.34 & $-0.7,0.02$ & 0.064 & 0.03 & $-0.25,0.31$ & 0.852 \\
\hline
\end{tabular}

GDM, gestational diabetes mellitus; GLT, glucose load test.

* A $P$ value $\leq 0.007$ was considered significant with Bonferroni correction.

† Generalised estimating equation was used to test the association between maternal factors and milk hormones with adjustment for maternal age.

\section{Breast milk hormone concentrations and infant growth}

The overall adiponectin concentration in BM during the first 3 months was inversely associated with the infant weight-for-height in both the GDM $(\beta=-2.49 ; 95 \% \mathrm{CI}-3 \cdot 83,-1 \cdot 15 ; P<0.001)$ and healthy groups $(\beta=-1.42 ; 95 \% \mathrm{CI}-2 \cdot 38,-0 \cdot 46 ; P=0.003$; Table 4). Adiponectin and insulin were associated with head circumference during the follow-up period in both the GDM $\left(\beta=-0.39 ; 95 \%\right.$ CI $-0 \cdot 65,-0 \cdot 13 ; P_{\text {adiponectin }}=0.003 ; \beta=-0.39$; $95 \%$ CI $\left.-0.65,-0.13 ; \quad P_{\text {insulin }}=0.004\right)$ and healthy groups $\left(\beta=-0.15 ; 95 \%\right.$ CI $-2 \cdot 38,-0 \cdot 46 ; P_{\text {adiponectin }}=0.007 ; \beta=-0.55$; $95 \%$ CI $-1 \cdot 11,0 \cdot 01 ; P_{\text {insulin }}=0.049$; Table 4). However, the association of insulin with head circumference in healthy subjects was insignificant after Bonferroni correction. We further plotted the BM hormone concentrations and weight-for-height 
Table 4. Associations of breast milk hormone concentrations with infant weight-for-height and head circumference ( $\beta$-Coefficients and $95 \%$ confidence intervals)

\begin{tabular}{|c|c|c|c|c|c|c|c|c|c|c|c|c|}
\hline \multirow[b]{3}{*}{ Milk hormones } & \multicolumn{6}{|c|}{ Weight-for-height } & \multicolumn{6}{|c|}{ Head circumference } \\
\hline & \multicolumn{3}{|c|}{ Exposed to GDM } & \multicolumn{3}{|c|}{ Unexposed to GDM } & \multicolumn{3}{|c|}{ Exposed to GDM } & \multicolumn{3}{|c|}{ Unexposed to GDM } \\
\hline & $\beta$ & $95 \% \mathrm{Cl}$ & $P^{\star}$ & $\beta$ & $95 \% \mathrm{Cl}$ & $P^{\star}$ & $\beta$ & $95 \% \mathrm{Cl}$ & $P^{\star}$ & $\beta$ & $95 \% \mathrm{Cl}$ & $P^{\star}$ \\
\hline Adiponectin ( $\mu \mathrm{g} / \mathrm{ml})$ & -2.49 & $-3.83,-1 \cdot 15$ & $<0.001$ & -1.42 & $-2.38,-0.46$ & 0.003 & -0.39 & $-0.65,-0.13$ & 0.003 & -0.15 & $-0.27,-0.03$ & 0.007 \\
\hline Leptin (ng/ml) & -0.01 & $-0.05,0.03$ & 0.687 & 0.01 & $-0.03,0.05$ & 0.572 & -0.001 & $-0.009,0.007$ & 0.797 & -0.01 & $-0.018,-0.002$ & 0.004 \\
\hline Insulin (U/ml) & -0.37 & $-1.73,0.99$ & 0.59 & $-1 \cdot 36$ & $-4 \cdot 46,1 \cdot 74$ & 0.383 & -0.39 & $-0.65,-0.13$ & 0.004 & -0.55 & $-1 \cdot 11,0.01$ & 0.049 \\
\hline Ghrelin (ng/ml) & -0.27 & $-0.85,0.31$ & 0.341 & 0.04 & $-0.1,0.18$ & 0.588 & 0.03 & $-0.09,0.15$ & 0.586 & -0.003 & $-0.017,0.011$ & 0.618 \\
\hline
\end{tabular}

GDM, gestational diabetes mellitus.

${ }^{*}$ A $P$ value $\leq 0.0125$ was considered significant with Bonferroni correction.

$\dagger$ In each generalised estimation equation model, we included weight-for-height as the dependent variable, and the hormone concentrations (i.e. ghrelin, adiponectin, leptin or insulin), gestational age and infant's sex as the independent variables.

at each time point to show the trend of the associations (online Supplementary Figure). The correlations shifted over time, which could be due to the differences in the concentrations between colostrum and mature milk.

\section{Discussion}

This study provides evidence supporting milk-borne adiponectin as an important nutritional mediator that links maternal metabolic status and early infant growth. Although women with GDM received dietary intervention or insulin therapy, adiponectin and ghrelin concentrations were decreased and insulin concentration was increased in their BM. Women with higher BMI had more adiponectin, leptin, insulin, but less ghrelin in BM. Among all the four hormones, adiponectin was inversely associated with early infant growth (i.e. weight-for-height and head circumference) in both women with GDM and healthy women, suggesting an independent modulatory role of milkborne adiponectin in infant growth.

The concentrations of adiponectin ${ }^{(26,36-38)}$ leptin $^{(18,37-39)}$ and insulin $^{(23)}$ in $\mathrm{BM}$ were in the same range comparing to prior studies. The total ghrelin concentration was comparable to that reported by Aydin who also used ELISA to quantify the hormones ${ }^{(22)}$. However, our read was ten times lower than the concentration measured using RIA, which could be due to the non-specific competition of proteins in $\mathrm{BM}^{(40)}$. To minimise the confounding effect of variation in the duration between milk delivery and collection ${ }^{(23)}$, we stored the BM samples immediately after collection. Other potential differences between GDM and healthy groups, including nulliparous mothers, maternal age, gestational age and delivery mode, were balanced between the GDM and healthy groups.

Women with controlled diabetes mellitus had normal milk lactose, glucose, protein, cholesterol, TAG and total fatty acid composition $^{(31)}$. However, their hormone concentrations varied and were associated with maternal BMI, glucose metabolism and the stage of lactation. Our results were consistent with prior studies that women with GDM or gravid hyperglycaemia had higher insulin ${ }^{(23)}$ and lower ghrelin concentration in their $\mathrm{BM}^{(33)}$. Higher BMI was associated with more leptin ${ }^{(18,41,42)}$, insulin $^{(23,43)}$ and less ghrelin in BM, which was due to the corresponding maternal hormone levels in serum ${ }^{(25)}$. Maternal BMI seemed to be a more important factor modulating hormone levels in BM. When maternal BMI were comparable in GDM and healthy groups on day 42 , all the four hormones were similar between groups. For adiponectin, prior studies and our study did not find a significant correlation between prepregnancy BMI and milk adiponectin ${ }^{(19,25)}$. Only two previous studies examined the association between post-pregnant BMI and adiponectin in $\mathrm{BM}^{(19,24)}$. Both prior and our study found positive association between maternal BMI and milk adiponectin. Maternal BMI fluctuated during lactation and lactation month was associated with both maternal BMI and milk components ${ }^{(24)}$. In our study, BMI during lactation was associated with BM adiponectin after accounting for time effect. It is noticeable that higher BMI was associated with a lower serum adiponectin concentration $^{(44)}$ and a higher $\mathrm{BM}$ adiponectin concentration. This inconsistency between serum and BM can be due to the modulatory function of prolactin, a major determinant of mammary gland secretion and a negative modulator for adiponectin secretion. Excess maternal adipose tissue can down-regulate the secretion of prolactin, and consequently increase the adiponectin concentration in $\mathrm{BM}^{(19)}$. Although GDM was associated with higher BMI, GDM seemed to decrease the adiponectin concentration in BM independently and more robustly than the effect of adipose tissue possibly by decreasing the circulating adiponectin.

Hormones in BM were suggested to protect infants from the short-term acceleration of adipose deposit and the long-term obesity and diabetes ${ }^{(45)}$. Prior studies in healthy infants and toddlers have shown that breastfed infants exposed to higher concentration of adiponectin in BM had a lower weight gain during the first year and a greater weight gain during the second year ${ }^{(24,30,42)}$. In Brunner's study, milk adiponectin concentration at week 6 tended to be inversely associated with infant anthropometry in the first 4 months, but was positively associated with infant weight gain and fat mass till 2 years of age. In the study by Woo et al., a higher median level of adiponectin across baseline (week 1), months 1, 3, 5 and 6 was associated with accelerated weight trajectory during the second year. We also found such an inverse association during the early infancy in children born to mothers with GDM. Our results partially support the hypothesis that $\mathrm{BM}$ adiponectin could mitigate early 
weight gain in infancy when fat mass gain is dominant, and it may promote weight gain in the second year of life when lean body mass is dominant ${ }^{(30)}$. $\mathrm{BM}$ adiponectin, thus, protects children against obesity in later life. With favourable controlled blood glucose, breast-feeding could help the infants of women with GDM gain growth trajectory comparable to that of infants born to healthy women ${ }^{(46)}$.

The strengths of this study include recruiting exclusively breastfed infants until $90 \mathrm{~d}$ before introduction of solid food, recruiting women with GDM under proper control to minimise the bias due to the effects of macronutrients in $\mathrm{BM}$, and drawing both foremilk and hindmilk. However, limitations should be noted when interpreting the results. First, based on prior studies demonstrating the association of hormones in maternal serum, $\mathrm{BM}$ and infant serum ${ }^{(47,48)}$, maternal and infant serum hormone concentration was not analysed in this study. Second, twentytwo dyads with GDM dropped out due to IGT, type 2 diabetes and introduction of formula feeding during the follow-ups, which may make the samples on 42 and $90 \mathrm{~d}$ less representative of the population with GDM. Finally, infant growth was evaluated using anthropometric measurements. More accurate measurements of body composition, such as MRI, will help accurately evaluate the mediation effect of hormones in BM between maternal metabolic status and infant growth and their protective effects on infants' metabolic health.

In conclusion, breastfed infants of women with controlled GDM gained normal growth trajectory. Milk-borne adiponectin could be an important nutritional mediator that links maternal metabolic status and early infant growth. It was decreased in BM of women with GDM and was associated with lower infant early weight gain. Further studies in infants fed by BM of women with diabetes, donor BM of healthy women, and formula are warranted to elucidate the role of milk-borne hormones as bioactive nutrients to attenuate the risk of childhood obesity associated with maternal diabetes.

\section{Acknowledgements}

The authors thank the parents who participated in the study. The authors also wish to thank Dr Simon Hugh Lam, Dr Albert Martin Li, Ms Yi Peng and Mr John Simmons Borchert for their review and advice on the manuscript.

This work was supported by grants from Beijing Municipal Science and Technology Commission (D111100000611001) and Beijing Science and Technology Star Program (2004A027). The sponsors did not influence the study design, analysis, interpretation of the results or writing of the manuscript

X. Y. contributed to conception and design of the study, follow-up of study subjects, data acquisition, hormone assays, data analysis and interpretation, drafting and critical revision of the manuscript and final approval of the manuscript for submission. S. S. R. was involved in data acquisition, skim milk preparation, data analysis and interpretation, drafting and critical revision of the manuscript and final approval of the manuscript for submission. X. S., G. D. and W. W. contributed to the design of the study, follow-up of study subjects and revision of the manuscript. L. Z. and S. W. participated in recruitment of subjects and sample collection. M. L. contributed to the design of the study, hormone assays, critical review of the manuscript and final approval of the manuscript for submission. D. W. was involved in conception and design of the study, critical review of the manuscript and final approval of the manuscript for submission.

The authors declare that there are no conflicts of interest.

\section{Supplementary material}

For supplementary material/s referred to in this article, please visit https://doi.org/10.1017/S0007114518002933

\section{References}

1. Correa A, Bardenheier B, Elixhauser A, et al. (2015) Trends in prevalence of diabetes among delivery hospitalizations, United States, 1993-2009. Matern Child Health J 19, 635-642.

2. World Health Organization (2017) Childhood Overweight and Obesity. http://www.who.int/gho/ncd/risk_factors/overweight_ obesity/obesity_adolescents/en/

3. Catalano PM, McIntyre HD, Cruickshank JK, et al. (2012) The hyperglycemia and adverse pregnancy outcome study: associations of GDM and obesity with pregnancy outcomes. Diabetes Care 35, 780-786.

4. Logan KM, Emsley RJ, Jeffries S, et al. (2016) Development of early adiposity in infants of mothers with gestational diabetes mellitus. Diabetes Care 39, 1045-1051.

5. Gillman MW, Rifas-Shiman S, Berkey CS, et al. (2003) Maternal gestational diabetes, birth weight, and adolescent obesity. Pediatrics 111, e221-e226.

6. Sellers EA, Dean HJ, Shafer LA, et al. (2016) Exposure to gestational diabetes mellitus: impact on the development of early-onset type 2 diabetes in Canadian First Nation and NonFirst Nation Offspring. Diabetes Care 39, 2240-2246.

7. Cripps RL, Archer ZA, Mercer JG, et al. (2007) Early life programming of energy balance. Biochem Soc Trans 35, 1203-1204.

8. Armstrong J \& Reilly JJ, Child Health Information Team (2002) Breastfeeding and lowering the risk of childhood obesity. Lancet 359, 2003-2004.

9. Owen CG, Martin RM, Whincup PH, et al. (2006) Does breastfeeding influence risk of type 2 diabetes in later life? A quantitative analysis of published evidence. Am J Clin Nutr 84, 1043-1054.

10. Plagemann A, Harder T, Franke K, et al. (2002) Long-term impact of neonatal breast-feeding on body weight and glucose tolerance in children of diabetic mothers. Diabetes Care $\mathbf{2 5}$, $16-22$.

11. Mayer-Davis EJ, Rifas-Shiman SL, Zhou L, et al. (2006) Breastfeeding and risk for childhood obesity: does maternal diabetes or obesity status matter? Diabetes Care 29, 2231-2237.

12. Crume TL, Ogden L, Maligie M, et al. (2011) Long-term impact of neonatal breastfeeding on childhood adiposity and fat distribution among children exposed to diabetes in utero. Diabetes Care 34, 641-645.

13. Savino F, Benetti S, Liguori SA, et al. (2013) Advances on human milk hormones and protection against obesity. Cell Mol Biol (Noisy-le-grand) 59, 89-98.

14. Bronsky J, Mitrova K, Nevoral J, et al. (2012) Immunoexpression of type-1 adiponectin receptor in the human intestine. Cesk Patol 48, 165-166.

15. Barrenetxe J, Villaro AC, Guembe L, et al. (2002) Distribution of the long leptin receptor isoform in brush border, basolateral membrane, and cytoplasm of enterocytes. Gut 50, 797-802. 
16. Andres SF, Simmons JG, Mah AT, et al. (2013) Insulin receptor isoform switching in intestinal stem cells, progenitors, differentiated lineages and tumors: evidence that IR-B limits proliferation. J Cell Sci 126, 5645-5656.

17. Mitrović O, Čokić V, Đikić D, et al. (2014) Ghrelin receptors in human gastrointestinal tract during prenatal and early postnatal development. Peptides 57, 1-11.

18. Schuster S, Hechler C, Gebauer C, et al. (2011) Leptin in maternal serum and breast milk: association with infants' body weight gain in a longitudinal study over 6 months of lactation. Pediatr Res 70, 633-637.

19. Martin LJ, Woo JG, Geraghty SR, et al. (2006) Adiponectin is present in human milk and is associated with maternal factors. Am J Clin Nutr 83, 1106-1111.

20. Fields DA \& Demerath EW (2012) Relationship of insulin, glucose, leptin, IL-6 and TNF-alpha in human breast milk with infant growth and body composition. Pediatr Obes $\mathbf{7}$, 304-312.

21. Dundar NO, Dundar B, Cesur G, et al. (2010) Ghrelin and adiponectin levels in colostrum, cord blood and maternal serum. Pediatr Int 52, 622-625.

22. Aydin S (2010) The presence of the peptides apelin, ghrelin and nesfatin-1 in the human breast milk, and the lowering of their levels in patients with gestational diabetes mellitus. Peptides 31, 2236-2240.

23. Ley SH, Hanley AJ, Sermer M, et al. (2012) Associations of prenatal metabolic abnormalities with insulin and adiponectin concentrations in human milk. Am J Clin Nutr 95, 867-874.

24. Woo JG, Guerrero ML, Altaye M, et al. (2009) Human milk adiponectin is associated with infant growth in two independent cohorts. Breastfeed Med 4, 101-109.

25. Andreas NJ, Hyde MJ, Gale C, et al. (2014) Effect of maternal body mass index on hormones in breast milk: a systematic review. PLOS ONE 9, e115043.

26. Weyermann M, Brenner H \& Rothenbacher D (2007) Adipokines in human milk and risk of overweight in early childhood: a prospective cohort study. Epidemiology 18, 722-729.

27. Doneray H, Orbak Z \& Yildiz L (2009) The relationship between breast milk leptin and neonatal weight gain. Acta Paediatr 98, 643-647.

28. Cesur G, Ozguner F, Yilmaz N, et al. (2012) The relationship between ghrelin and adiponectin levels in breast milk and infant serum and growth of infants during early postnatal life. J Physiol Sci 62, 185-190.

29. Chan D, Goruk S, Becker AB, et al. (2017) Adiponectin, leptin and insulin in breast milk: associations with maternal characteristics and infant body composition in the first year of life. Int J Obes (Lond) 42, 36-43.

30. Woo JG, Guerrero ML, Guo F, et al. (2012) Human milk adiponectin affects infant weight trajectory during the second year of life. J Pediatr Gastroenterol Nutr 54, 532-539.

31. van Beusekom CM, Zeegers TA, Martini IA, et al. (1993) Milk of patients with tightly controlled insulin-dependent diabetes mellitus has normal macronutrient and fatty acid composition. Am J Clin Nutr 57, 938-943.

32. American Diabetes Association (2014) Diagnosis and classification of diabetes mellitus. Diabetes Care 37, Suppl. 1, S81-S90.

33. Aydin S, Geckil H, Karatas F, et al. (2007) Milk and blood ghrelin level in diabetics. Nutrition 23, 807-811.

34. Machin D, Campbell M, Fayers P, et al. (1997) Sample Size Tables for Clinical Studies, 2nd ed. Malden, MA: Blackwell Science.

35. Neter J, Wasserman W \& Kutner M (1983) Applied Linear Regression Models. Chicago, IL: Richard D. Irwin Inc.

36. Newburg DS, Woo JG \& Morrow AL (2010) Characteristics and potential functions of human milk adiponectin. J Pediatr 156, S41-S46.

37. Bronsky J, Mitrova K, Karpisek M, et al. (2011) Adiponectin, AFABP, and leptin in human breast milk during 12 months of lactation. J Pediatr Gastroenterol Nutr 52, 474-477.

38. Savino F, Liguori SA, Sorrenti M, et al. (2011) Breast milk hormones and regulation of glucose homeostasis. Int J Pediatr 2011, 803985

39. Savino F, Liguori SA, Petrucci E, et al. (2010) Evaluation of leptin in breast milk, lactating mothers and their infants. Eur J Clin Nutr 64, 972-977.

40. Prudom C, Liu J, Patrie J, et al. (2010) Comparison of competitive radioimmunoassays and two-site sandwich assays for the measurement and interpretation of plasma ghrelin levels. I Clin Endocrinol Metab 95, 2351-2358.

41. Schueler J, Alexander B, Hart AM, et al. (2013) Presence and dynamics of leptin, GLP-1, and PYY in human breast milk at early postpartum. Obesity (Silver Spring) 21, 1451-1458.

42. Brunner S, Schmid D, Zang K, et al. (2015) Breast milk leptin and adiponectin in relation to infant body composition up to 2 years. Pediatr Obes 10, 67-73.

43. Ahuja S, Boylan M, Hart S, et al. (2011) Glucose and insulin levels are increased in obese and overweight mothers' breastmilk. Food Nutr Sci 2, 201-206.

44. Cnop M, Havel PJ, Utzschneider KM, et al. (2003) Relationship of adiponectin to body fat distribution, insulin sensitivity and plasma lipoproteins: evidence for independent roles of age and sex. Diabetologia 46, 459-469.

45. Savino F, Fissore MF, Liguori SA, et al. (2009) Can hormones contained in mothers' milk account for the beneficial effect of breast-feeding on obesity in children? Clin Endocrinol (Oxf) 71, 757-765.

46. Whitmore TJ, Trengove NJ, Graham DF, et al. (2012) Analysis of insulin in human breast milk in mothers with type 1 and type 2 diabetes mellitus. Int J Endocrinol 2012, 296368.

47. Savino F, Lupica M, Benetti S, et al. (2012) Adiponectin in breast milk: relation to serum adiponectin concentration in lactating mothers and their infants. Acta Paediatr 101, 1058-1062.

48. Weyermann M, Beermann C, Brenner H, et al. (2006) Adiponectin and leptin in maternal serum, cord blood, and breast milk. Clin Chem 52, 2095-2102. 